PROCEEDINGS OF THE

AMERICAN MATHEMATICAL SOCIETY

Volume 139, Number 1, January 2011, Pages 219-230

S 0002-9939(2010)10468-9

Article electronically published on July 8, 2010

\title{
SOLUTIONS FOR NONLINEAR ELLIPTIC EQUATIONS WITH GENERAL WEIGHT IN THE SOBOLEV-HARDY SPACE
}

\author{
YIMIN ZHANG, JUN YANG, AND YAOTIAN SHEN
}

(Communicated by Yingfei Yi)

\begin{abstract}
In this paper we apply Morse theory to study the existence of nontrivial solutions for nonlinear elliptic equations with general weight and Hardy potential in the Sobolev-Hardy space.
\end{abstract}

\section{INTRODUCTION}

This paper is motivated by the recent interest in elliptic equations of the form

$$
\left\{\begin{array}{l}
-\operatorname{div}(\phi \nabla u)-\psi u=f(x, u), \quad x \in \Omega, \\
u=0, \quad x \in \partial \Omega,
\end{array}\right.
$$

where $0 \in \Omega$ is a bounded domain in $\mathbb{R}^{N}, \phi$ is a positive continuous function, for a positive continuous function $h$ satisfies $r^{N-1} \phi(r)\left(h^{2}(r)\right)^{\prime}=c_{0}$ for some constant $c_{0}$, and $h^{-1}(0)=0, \psi=\phi\left(-\frac{h^{\prime}}{h}\right)^{2}$ is the Hardy potential.

In this paper, we denote the Sobolev-Hardy space $H_{0,1}^{1}(\Omega)$ by the completion of $C_{0}^{\infty}(\Omega)$ with respect to the norm

$$
\|u\|_{1,1, \phi}=\left[\int_{\Omega}\left(\phi|\nabla u|^{2}-\psi u^{2}\right) d x\right]^{1 / 2},
$$

whose corresponding inner product is

$$
(u, \varphi)_{1,1, \phi}=\int_{\Omega}(\phi \nabla u \nabla \varphi-\psi u \varphi) d x .
$$

Note that $H_{0,1}^{1}(\Omega)$ is a Hilbert space. Also, we denote that $H=H_{0,1}^{1}(\Omega)$ and $\|u\|=\|u\|_{1,1, \phi}$. Then the corresponding functional of (0.1) is defined by

$$
I(u)=\frac{1}{2}\|u\|^{2}-\int_{\Omega} F(x, u) d x,
$$

where $F(x, s)=\int_{0}^{s} f(x, t) d t$.

Received by the editors November 19, 2009 and, in revised form, February 26, 2010

2010 Mathematics Subject Classification. Primary 35J65, 35J40.

Key words and phrases. Morse theory, the PSC condition, critical groups, Sobolev-Hardy space.

The project was supported in part by the National Natural Science Foundation of China (10771074), the NNSF of China (No. 10801055) and the Doctoral Program of NEM of China (No. 200805611026).

(C)2010 American Mathematical Society Reverts to public domain 28 years from publication 
Denote the function $\bar{h}$ by

$$
\bar{h}\left(r_{1}, r_{2}\right)=c_{0} \int_{r_{1}}^{r_{2}} \phi^{-1} r^{1-N} d r
$$

for $0 \leq r_{1} \leq r_{2} \leq \infty$. Let us consider the following two possible cases:

$\left(A_{1}\right) \bar{h}(r, \infty)<\infty$ for all $r>0$ and $\bar{h}^{-1}(0, \infty)=0$;

$\left(A_{2}\right) \bar{h}(r, D)<\infty$ and $\bar{h}^{-1}(0, D)=0$ for some $D>\sup _{x \in \Omega}|x|$.

Denote the function $h_{1}$ by

$$
h_{1}^{2}(r)= \begin{cases}\frac{2}{c_{0}} \ln \frac{h(r)}{h(D)}, & \text { if }\left(A_{1}\right) \text { holds, } \\ \frac{2}{c_{0}} \ln h(r), & \text { if }\left(A_{2}\right) \text { holds } .\end{cases}
$$

Before stating our results, let us recall the eigenvalue problem

$$
\left\{\begin{array}{l}
-\operatorname{div}(\phi \nabla u)-\psi u=\lambda \psi_{1} u, \quad x \in \Omega, \\
u=0, \quad x \in \partial \Omega,
\end{array}\right.
$$

where $\psi_{1}=\phi\left(\frac{h_{1}^{\prime}}{h_{1}}\right)^{2}$. The first eigenvalue of problem (0.6) is

$$
\lambda_{1}\left(\phi, \psi_{1}\right)=\inf \left\{\|u\|^{2} \mid \int_{\Omega} \psi_{1} u^{2} d x=1, \quad u \in H\right\},
$$

which is simple and isolated and has a positive eigenfunction $u_{1}$. Similarly to [14, we denote the eigenvalue sequence of problem (0.6) by

$$
\begin{aligned}
\lambda_{n}\left(\phi, \psi_{1}\right)=\inf \left\{\|u\|^{2} \mid \int_{\Omega} \psi_{1} u^{2} d x=1, \int_{\Omega} \psi_{1} u u_{i} d x=0\right. \\
\quad \text { for } i=1,2, \cdots, n-1, u \in H\} .
\end{aligned}
$$

Assume the Carathéodory function $f(x, s)$ satisfies:

$\left(f_{1}\right)|f(x, s)| \leq C \psi_{1}|s|+C \theta|s|^{p-1}$ for some $2<p<2^{*}$, where

$$
\theta=r^{1-N} h^{-(1+p)}\left(-h^{\prime}\right) h_{1}^{-(2+p)} .
$$

$\left(f_{2}\right) \limsup _{s \rightarrow 0} \frac{f(x, s)}{\psi_{1} s}=\nu$ uniformly for a.e. $x \in \Omega$.

$\left(f_{3}\right) \limsup _{s \rightarrow \infty} \frac{f(x, s)}{\psi_{1} s}=\mu$ uniformly for a.e. $x \in \Omega$.

$\left(f_{4}\right)$ There exist $\beta \geq 1$ such that $\beta H(x, s) \geq H(x, t s)$, for $(x, s) \in \Omega \times \mathbb{R}$ and $t \in[0,1]$, where $H(x, s)=s f(x, s)-2 F(x, s)$.

$\left(f_{5}\right)$ There exist $\sigma \in(0,2)$ and $\alpha$ a nonpositive constant, such that

$$
\liminf _{|s| \rightarrow 0} \frac{\mu F(x, s)-s f(x, s)}{\psi_{1}|s|^{2}} \geq \alpha>\lambda_{1}\left(\phi, \psi_{1}\right)\left(\frac{\sigma}{2}-1\right) \text {, uniformly a.e. } x \in \Omega \text {. }
$$

When $\psi=0$, Caldiroli and Musina [5] studied problem (0.1) with $\phi(x) \geq$ $C\left|x-x_{0}\right|^{\alpha}, 0<\alpha<2, x \in B_{r}\left(x_{0}\right) \subset \Omega$. In addition, they considered the case of finite many singular points as $x_{0}$. When $\phi=1$ and $\psi=\kappa q(x) /|x|^{2}$ with $\kappa<(N-2)^{2} / 4$, problem (0.1) has been discussed in many papers such as [9, 10]. Adimurthi and Sandeep [3] studied the existence or nonexistence of the solution when $\kappa=(N-2)^{2} / 4$ and $f(x, s)=\vartheta \eta(x) s$. If $\kappa>(N-2)^{2} / 4$, then the operator $-\Delta-\frac{\mu}{|x|^{2}}$ is unbounded; see [10]. It has been proved in 2] that problem (0.1) has solutions in the sense of distribution when $\psi(x)<\frac{1}{|x|^{2}}$, and it has no solution in $H_{0}^{1}(\Omega)$ when $\psi(x)=\frac{1}{|x|^{2}}$. However, Shen and Yao [17] proved the existence of a solution in a new Hilbert space when $\psi(x)=\frac{1}{|x|^{2}}$ and $f(x, s)$ is superlinear. When 
problem (0.1) has a general weight function $\phi(|x|)$, only a few existence results have been proved so far by means of techniques of critical point theory. We can mention Shen and Chen [14, who were the first to consider the degenerate elliptic equation with a general weight and Hardy potential.

If $f(x, 0)=0$, then problem $(0.1)$ has a trivial solution $u=0$. We are interested in finding nontrivial solutions of problem (0.1). It follows from Morse theory that comparing the critical groups of $I$ at zero and infinity may yield the existence of nontrivial solutions to problem (0.1) (cf. [6]). The critical groups depend mainly upon the behaviors of the function $f(x, s)$ or its primitive $F(x, s)$ near zero and near infinity, respectively. In this paper, we want to study the existence of nontrivial solutions of problem (0.1) by using Morse theory and imposing various conditions on $f(x, s)$ or its primitive $F(x, s)$ near the origin and near infinity.

Our main results are the following:

Theorem 0.1. Assume that function $f(x, s)$ satisfies $\left(f_{1}\right),\left(f_{2}\right)$ with $\nu \in\left(0, \lambda_{1}\left(\phi, \psi_{1}\right)\right)$ and one of the following conditions:

(1) $\left(f_{3}\right)$ with $\mu \in\left(\lambda_{m}\left(\phi, \psi_{1}\right), \lambda_{m+1}\left(\phi, \psi_{1}\right)\right)$ and $m>0$.

(2) $\left(f_{3}\right)$ with $\mu=\infty$ and $\left(f_{4}\right)$.

Then problem (0.1) has at least one nontrivial weak solution in $H$.

Remark. In this theorem, condition $\left(f_{2}\right)$ is a nonresonance condition. Condition (2) was studied in [14 by using the mountain pass theorem. In this paper, we consider it by using Morse theory. Moreover we consider it asymptotically linear in the infinity as in case (1).

Theorem 0.2. Assume that function $f(x, s)$ satisfies $\left(f_{1}\right),\left(f_{2}\right)$ with $\nu \in\left(\lambda_{l}\left(\phi, \psi_{1}\right)\right.$, $\left.\lambda_{l+1}\left(\phi, \psi_{1}\right)\right)$ and one of the following conditions:

(1) $\left(f_{3}\right)$ with $\mu \in\left(\lambda_{m}\left(\phi, \psi_{1}\right), \lambda_{m+1}\left(\phi, \psi_{1}\right)\right)$ and $m \neq l$.

(2) $\left(f_{3}\right)$ with $\mu=\infty$ and $\left(f_{4}\right)$.

Then problem (0.1) has at least one nontrivial weak solution in $H$.

Remark. This time, condition $\left(f_{2}\right)$ means that problem $(0.1)$ is resonant near zero at the eigenvalue $\lambda_{l}\left(\phi, \psi_{1}\right)$ from the right side. We also do not need to assume that the limit exists, and we can replace $\left(f_{2}\right)$ by $\lambda_{l}\left(\phi, \psi_{1}\right)|s|^{2} \leq 2 F(x, s) \leq \mu|s|^{2}$, for $x \in$ $\Omega,|s| \leq r$, where $r>0$. Condition (1) is considered the first time by using Morse theory. When $\phi=1$ and $\psi=0$, condition (2) was studied in 14, Theorem 1.1, but in the infinity they assume the superquadraticity condition: There exist $M>0$ and $\theta>2$ such that

$$
0<\theta F(x, s) \leq s f(x, s) \text { for } x \in \Omega,|s| \geq M .
$$

In this case, we weaken the superquadraticity condition to $\left(f_{3}\right)$ and $\left(f_{4}\right)$.

Theorem 0.3. Assume that function $f(x, s)$ satisfies $\left(f_{1}\right),\left(f_{5}\right),\left(f_{2}\right)$ with $\nu=\infty$ and $\left(f_{3}\right)$ with $\mu \in\left(\lambda_{m}\left(\phi, \psi_{1}\right), \lambda_{m+1}\left(\phi, \psi_{1}\right)\right)$ for $m \geq 0$. Then problem (0.1) has at least one nontrivial weak solution in $H$.

Remark. When $\phi=1$ and $\psi=0$, problem (0.1) was considered in [8, Theorem 1.5, under the conditions $\left(f_{1}\right),\left(f_{2}\right)$ with $\nu=\infty,\left(f_{3}\right)$ and

$$
2 F(x, s)-s f(x, s)>0 \text { for all } x \in \Omega \text { and } t \neq 0 .
$$

Condition (0.7) is a strong assumption; we drop it and assume $\left(f_{5}\right)$. 
As we have mentioned, we will use Morse theory to prove our main existence results. Let us now collect some concepts and results that will be used below. We denote by $K$ the set of critical points of $I$,

$$
K:=\left\{u \in X: I^{\prime}(u)=0\right\},
$$

and let

$$
K_{c}:=\left\{u \in X: I^{\prime}(u)=0, I(u)=c\right\} .
$$

If $K_{c} \neq \emptyset$, the real number $c$ is called a critical value of $I$; otherwise, it is called a regular value of $I$. If $u \in K_{c}$ and for all $\delta>0, B(u ; \delta) \cap K_{c}=u$, then we call $u$ an isolated critical point. Let

$$
I^{c}:=\{u \in X: I(u)<c\}
$$

denote an open sub-level set of $I$.

Given a pair $(A, B)$ in the metric space $X$, let $H_{q}(A, B)$ denote the $q$ th relative singular homology group of the pair $(A, B)$, with coefficients in $\mathbb{Z}$.

Definition 0.4 ([6]). Let $I: X \rightarrow \mathbb{R}$ be a continuous function. For $u \in X$, $I(u)=c, U$ a neighborhood of $u$ and $q$ a nonnegative integer, set

$$
C_{q}(I ; u)=H_{q}\left(\left(I^{c} \cup u\right) \cap U, I^{c} \cap U\right) .
$$

Then we call the vector space $C_{q}(I ; u)$ for the $q$ th critical group of $I$ at $u$.

Remark. Due to the excision property of singular homology [6], the definition of the critical groups does not depend on the particular choice of the neighborhood $U$. If the functional $I$ satisfies the PSC condition and if for $[a, b] \subset \mathbb{R} \cup\{\infty\}$ the critical set

$$
K_{a}^{b}=\left\{u \in H_{0}^{1}(\Omega) ; a \leq I(u) \leq b, I^{\prime}(u)=0\right\}
$$

is finite, we have the following Morse relations between the Morse critical groups and homological characterization of subset sets:

$$
H_{q}\left(I^{b}, I^{a}\right)=\bigoplus_{K_{a}^{b}} C_{q}(I ; u) .
$$

Let $a<\inf I(K)$. We refer to the critical group of $I$ at infinity as $C_{q}(I ; \infty):=$ $H_{q}\left(X, I^{a}\right), q \in \mathbb{Z}$ (cf. [11], 13]).

Definition 0.5. The functional $I$ satisfies $\left(D_{c}\right)$ at the level $c \in \mathbb{R}$ if for any $\bar{\varepsilon}>0$ and any neighborhood $\mathcal{N}$ of $K_{c}$, there are $\varepsilon>0$ and a continuous deformation $\eta: X \times[0,1] \rightarrow X$, such that

(1) $\eta(u, t)=u$ for either $t=0$ or $u \bar{\epsilon} I^{-1}[c-\bar{\varepsilon}, c+\bar{\varepsilon}]$;

(2) $I(\eta(u, t))$ is nonincreasing in $t$ for any $u \in X$;

(3) $\eta\left(I^{c+\varepsilon} \backslash \mathcal{N}\right) \subset I^{c-\varepsilon}$.

$I$ satisfies $(D)$ if $I$ satisfies $\left(D_{c}\right)$ for all $c \in \mathbb{R}$.

Definition 0.6. A functional $I \in C^{1}(X, \mathbb{R})$ is said to satisfy the PSC condition in $\left(d_{1}, d_{2}\right)\left(-\infty \leq d_{1}<d_{2} \leq+\infty\right)$ if

(1) every bounded sequence $\left\{u_{n}\right\}$ such that $\left\{I\left(u_{n}\right)\right\}$ is bounded and $I^{\prime}\left(u_{n}\right) \rightarrow 0$ possesses a convergent subsequence, and

(2) $\forall c \in\left(d_{1}, d_{2}\right)$ there are $R, a>0$, such that $[c-\sigma, c+\sigma] \subset\left(d_{1}, d_{2}\right)$ and any $u \in I^{-1}([c-\sigma, c+\sigma])$ with $\|u\| \geq R$ satisfies $\left\|I^{\prime}(u)\right\|\|u\| \geq a$. 
Remark. We note that $I$ satisfies the deformation condition if $I$ satisfies the PSC condition. Let $I$ satisfy the $(D)$ condition, and let $u=0$ be a critical point of $I$. Morse theory [6], 12] tells us that if $K=\{0\}$, then $C_{q}(I ; \infty) \cong C_{q}(I ; 0)$ for all $q \in \mathbb{Z}$. It follows that if $C_{q}(I ; \infty) \neq C_{q}(I ; 0)$ for some $q \in \mathbb{Z}$, then $I$ must have a nontrivial critical point.

\section{Preliminary Results}

Lemma 1.1 (14, Theorem 1.4). Let $N>2,0 \in \Omega \subset B_{D}(0)$ and $\phi \in C(0, D)$ satisfy $\left(A_{1}\right)$ or $\left(A_{2}\right)$. If

$$
\left|\frac{\phi^{\prime}}{\phi}\right| \leq M_{1}\left|\frac{h^{\prime}}{h}\right| \leq M_{2} r^{-1}, \forall r \in(0, D),
$$

for some positive constants $M_{1}$ and $M_{2}$, then there exists a constant $C>0$ such that for any $u \in H$,

$$
\|u\|^{2} \geq C\left(\int_{\Omega} \eta|u|^{2^{*}} d x\right)^{2 / 2^{*}}
$$

where $\eta=r^{1-N} h^{-\left(1+2^{*}\right)} h_{1}^{-\left(2+2^{*}\right)}$.

Lemma 1.2 ([15], Theorem 1.3). Let $N>2,0 \in \Omega \subset B_{D}(0)$ and $\phi \in C(0, D)$ satisfy $\left(A_{1}\right)$ or $\left(A_{2}\right)$. There exists a constant $C>0$ such that for any $u \in H$,

$$
\|u\|^{2} \geq C \int_{\Omega} \psi_{1} u^{2} d x .
$$

Remark. When $\phi=1$, Adimurthi et al. [1 obtained the inequalities (1.3) in $W_{0}^{1, p}(\Omega)$. A series of results in this aspect was given by Barbatis et al. in 4 .

Lemma 1.3 (14, Theorem 1.6). Let $N>2,0 \in \Omega \subset B_{D}(0)$ and $\phi \in C(0, D)$ satisfy $\left(A_{1}\right)$ or $\left(A_{2}\right)$, for any $2<p<2^{*}$. Then

$$
\begin{aligned}
& \left(\int_{\Omega} \theta|u|^{p} d x\right)^{1 / p} \leq\left(\int_{\Omega} \psi_{1}|u|^{2} d x\right)^{\lambda / 2}\left(\int_{\Omega} \eta|u|^{2^{*}} d x\right)^{(1-\lambda) / 2}, \\
& \left(\int_{\Omega} \theta|u|^{p} d x\right)^{1 / p} \leq C\left(\int_{\Omega} \psi_{1}|u|^{2} d x\right)^{\lambda / 2}\|u\|^{(1-\lambda) / 2}
\end{aligned}
$$

for any $u \in H$, where $\frac{1}{p}=\frac{\lambda}{2}+\frac{1-\lambda}{2^{*}}$ and

$$
\theta=\psi_{1}^{p \lambda / 2} \eta^{p(1-\lambda) / 2^{*}}=r^{1-N} h^{-(1+p)}\left(-h^{\prime}\right) h_{1}^{-(2+p)} .
$$

Lemma 1.4. Assume $\left(f_{1}\right)$ and $\left(f_{3}\right)$ with $\mu<\infty$ is not an eigenvalue of problem (0.6). Then the functional I satisfies the Palais-Smale (PS) condition.

Proof. Since the function $f(x, s)$ satisfies the subcritical growth condition $\left(f_{1}\right)$, a standard argument (cf. [14, [16]) shows that we just have to prove that the PS sequence is bounded. Assume it does not hold. Then there exists a sequence $\left\{u_{n}\right\}$, $\left\|u_{n}\right\| \rightarrow \infty$ and $c \in \mathbb{R}$ such that for any $\varphi \in H$,

$$
\begin{gathered}
\frac{1}{2}\left\|u_{n}\right\|^{2}-\int_{\Omega} F\left(x, u_{n}\right) d x \rightarrow c, \\
\int_{\Omega} \phi \nabla u_{n} \nabla \varphi d x-\int_{\Omega} \psi u_{n} \varphi d x-\int_{\Omega} f\left(x, u_{n}^{+}\right) \varphi d x \rightarrow 0 .
\end{gathered}
$$


Set $v_{n}=u_{n} /\left\|u_{n}\right\|$. Then $\left\|v_{n}\right\|=1$, and let $v$ be such that (up to $q$ subsequence)

$$
v_{n} \rightarrow v \text { in } H ; v_{n} \rightarrow v \text { in } L^{2}(\Omega) ; \quad v_{n} \rightarrow v \text { a.e. } x \in \Omega \text {. }
$$

Multiplying both side of (1.7) by $1 /\left\|u_{n}\right\|$ we have

$$
\int_{\Omega} \phi \nabla v_{n} \nabla \varphi d x-\int_{\Omega} \psi v_{n} \varphi d x-\int_{\Omega} \frac{f\left(x, u_{n}^{+}\right)}{\left\|u_{n}\right\|} \varphi d x \rightarrow 0 .
$$

If $v(x)>0$, then from $\left(f_{3}\right)$ we have

$$
\frac{f\left(x, u_{n}^{+}\right)}{\left\|u_{n}\right\|} \varphi=\frac{f\left(x, u_{n}^{+}\right)}{u_{n}^{+}} v_{n}^{+} \varphi \rightarrow \mu \psi_{1} v^{+} \varphi, \text { a.e. } x \in \Omega,
$$

for all $\varphi \in H$. At the same time, from $\left(f_{3}\right)$ there exists a constant $C>0$ such that

$$
\left|\frac{f\left(x, u_{n}^{+}\right)}{u_{n}^{+}} v_{n}^{+} \varphi\right| \leq C \mu \psi_{1} v_{n}^{+}|\varphi| .
$$

Since $\mu \psi_{1} v_{n}^{+} \varphi \rightarrow \mu \psi_{1} v^{+} \varphi$, from (a variant of) Lebesgue's dominated convergence theorem we deduce that

$$
\int_{\Omega} \frac{f\left(x, u_{n}^{+}\right)}{u_{n}^{+}} v_{n}^{+} \varphi d x \rightarrow \mu \int_{\Omega} \psi_{1} v^{+} \varphi d x .
$$

Similarly we can deduce that

$$
\int_{\Omega} \frac{2 F\left(x, u_{n}^{+}\right)}{\left(u_{n}^{+}\right)^{2}}\left(v_{n}^{+}\right)^{2} d x \rightarrow \mu \int_{\Omega} \psi_{1}\left(v^{+}\right)^{2} d x .
$$

From (1.9) and (1.10), we have

$$
\int_{\Omega} \phi \nabla v_{n} \nabla \varphi d x-\int_{\Omega} \psi v_{n} \varphi d x-\mu \int_{\Omega} \psi_{1} v^{+} \varphi d x \rightarrow 0 .
$$

From (1.6) and (1.11), we have

$$
\int_{\Omega} \phi\left|\nabla v_{n}\right|^{2} d x-\int_{\Omega} \psi v_{n}^{2} d x-\mu \int_{\Omega} \psi_{1}\left(v^{+}\right)^{2} d x \rightarrow 0 .
$$

From (1.12) (taking $\varphi=v$ ) and (1.13), it is easy to deduce that $v_{n} \rightarrow v$ in $H$. Then, taking $n \rightarrow \infty$ in (1.12), we have

$$
\int_{\Omega} \phi \nabla v \nabla \varphi d x-\int_{\Omega} \psi v \varphi d x=\mu \int_{\Omega} \psi_{1} v^{+} \varphi d x,
$$

which is a contradiction since $\mu$ is not the eigenvalue of problem (0.6).

Lemma 1.5 ([14, Lemma 2.3). Assume $\left(f_{4}\right)$ and $\left(f_{3}\right)$ with $\mu=\infty$. Then the functional $I(u)$ satisfies the PSC condition.

\section{Critical group in the origin}

Lemma 2.1. Assume $\left(f_{1}\right)$ and $\left(f_{2}\right)$ with $\nu \in\left(0, \lambda_{1}\left(\phi, \psi_{1}\right)\right)$. Then $C_{q}(I ; 0) \cong \delta_{q, 0} \mathbb{Z}$ for all $q \in \mathbb{Z}$.

Proof. For any $u \in H$, it follows from $\left(f_{1}\right)$ and $\left(f_{2}\right)$ that

$$
F(x, s) \leq \frac{1}{2} \nu \psi_{1}|s|^{2}+C \theta|s|^{p}
$$


for any $2<p<2^{*}$. Then from Lemma 1.2 and Lemma 1.3 we have

$$
\begin{aligned}
I(u) & =\frac{1}{2}\|u\|^{2}-\int_{\Omega} F(x, u) d x \\
& \geq \frac{1}{2}\|u\|^{2}-\frac{1}{2} \nu \int_{\Omega} \psi_{1}|u|^{2} d x-C \int_{\Omega} \theta|u|^{p} d x \\
& \geq \frac{1}{2}\left(1-\frac{\nu}{\lambda_{1}\left(\phi, \psi_{1}\right)}\right)\|u\|^{2}-C\|u\|^{p} .
\end{aligned}
$$

Hence, $u=0$ is a local minimizer of $I(u)$. From [6], we have

$$
C_{q}(I ; 0) \cong \delta_{q ; 0} \mathbb{Z} \text { for all } q \in \mathbb{Z}
$$

Lemma 2.2. Assume $\left(f_{1}\right)$ and $\left(f_{2}\right)$ with $\nu \in\left(\lambda_{l}\left(\phi, \psi_{1}\right), \lambda_{l+1}\left(\phi, \psi_{1}\right)\right)$. Then $C_{l}(I ; 0)$ $\neq 0$.

Proof. Assume $W=\operatorname{span}\left\{u_{1}, u_{2}, \cdots, u_{l}\right\}$, where $u_{i}$ denotes the $i$ th eigenfunction of problem (0.6). Then $H=W \bigoplus W^{\perp}$. In fact, if $u \in W^{\perp}$, then

$$
\|u\|^{2} \geq \lambda_{l+1}\left(\phi, \psi_{1}\right) \int_{\Omega} \psi_{1} u^{2} d x
$$

Hence

$$
\begin{aligned}
I(u)= & \frac{1}{2}\|u\|^{2}-\int_{\Omega} F(x, u) d x \\
= & \frac{1}{2}\|u\|^{2}-\frac{1}{2} \nu \int_{\Omega} \psi_{1} u^{2} d x-\int_{\Omega}\left[F(x, u)-\frac{1}{2} \nu \psi_{1} u^{2}\right] d x \\
\geq & \frac{1}{2} C\left(1-\frac{\nu}{\lambda_{l+1}\left(\phi, \psi_{1}\right)}\right)\|u\|^{2}+\int_{|u| \leq r}\left[\frac{1}{2} \nu \psi_{1} u^{2}-F(x, u)\right] d x \\
& +\int_{|u|>r}\left[\frac{1}{2} \nu \psi_{1} u^{2}-F(x, u)\right] d x \\
\geq & \frac{1}{2} C\left(1-\frac{\nu}{\lambda_{l+1}\left(\phi, \psi_{1}\right)}\right)\|u\|^{2}-\int_{|u|>r} C \theta|u|^{p} d x \\
\geq & \frac{1}{2} C\left(1-\frac{\nu}{\lambda_{l+1}\left(\phi, \psi_{1}\right)}\right)\|u\|^{2}-C\|u\|^{p}
\end{aligned}
$$

for all $2<p<2^{*}$. It follows that if $u \in W^{\perp}$ and $0<\|u\|<\rho$ for $\rho>0$ small, then

$$
I(u)>0 .
$$

On the other hand, take $u \in W$; then $u=\sum_{i=1}^{l} a_{i} u_{i}$. Since $W$ is finite dimensional, it is easily seen that $\|u\| \leq \rho \Rightarrow|u| \leq r, \forall x \in \Omega$ for $\rho>0$ small and $r>0$. 
So it follows from $\left(f_{2}\right)$ and $\nu \in\left(\lambda_{l}\left(\phi, \psi_{1}\right), \lambda_{l+1}\left(\phi, \psi_{1}\right)\right)$ that for $\|u\| \leq \rho$,

$$
\begin{aligned}
I(u) & =\frac{1}{2}\|u\|^{2}-\int_{\Omega} F(x, u) d x \\
& =\frac{1}{2}\left\|\sum_{i=1}^{l} a_{i} u_{i}\right\|^{2}-\int_{\Omega} F(x, u) d x \\
& =\frac{1}{2} \sum_{i=1}^{l} a_{i}^{2} \lambda_{i}\left(\phi, \psi_{1}\right) \int_{\Omega} \psi_{1} u_{i}^{2} d x-\int_{\Omega} F(x, u) d x \\
& <\frac{1}{2} \lambda_{l}\left(\phi, \psi_{1}\right) \int_{\Omega} \psi_{1} u^{2} d x-\int_{\Omega} F(x, u) d x \\
& =\int_{|u|<r}\left[\frac{1}{2} \lambda_{l}\left(\phi, \psi_{1}\right) \psi_{1} u^{2}-F(x, u)\right] d x \leq 0 .
\end{aligned}
$$

Notice that $\operatorname{dim} W=l$. It follows from [13], Proposition 2.1, that

$$
C_{l}(I ; 0) \neq 0 \text {. }
$$

Lemma 2.3. Assume $\left(f_{1}\right),\left(f_{5}\right)$ and $\left(f_{2}\right)$ with $\nu=\infty$. Then $C_{q}(I ; 0) \cong 0$ for all $q \in \mathbb{Z}$.

Proof. For $0 \neq u \in H$ and $\left(f_{2}\right)$ we have

$$
\begin{aligned}
\frac{I(t u)}{t^{2}} & =\frac{1}{2}\|u\|^{2}-\int_{\Omega} \frac{F(x, t u)}{t^{2}} d x \\
& =\frac{1}{2}\|u\|^{2}-\int_{\Omega} \frac{F(x, t u)}{(t u)^{2}} u^{2} d x \rightarrow-\infty, \text { as } t \rightarrow 0 .
\end{aligned}
$$

Then there exists an $t_{0}>0$ such that

$$
I(t u)<0, \text { for all } 0<t<t_{0}
$$

Indeed, let $u \in H$ be such that $I(u)=0$; that is,

$$
\|u\|^{2}=2 \int_{\Omega} F(x, u) d x .
$$

In turn, for $\left(f_{1}\right)$ and $\left(f_{5}\right)$ respectively, we have for $\varepsilon>0$ sufficiently small that there exists $r=r(\varepsilon)>0$ such that

$$
\mu F(x, u)-u f(x, u) \geq(\alpha-\varepsilon) \psi_{1}|u|^{2}, \text { a.e. } x \in \Omega \text { and }|u| \leq r
$$

and

$$
\mu F(x, u)-u f(x, u) \geq-c_{\varepsilon} \theta|u|^{p} \text {, a.e. } x \in \Omega \text { and }|u|>r,
$$

for some $2<p<2^{*}$ and $c_{\varepsilon}>0$. 
From (2.2)-(2.4), using Lemma 1.2 and Lemma 1.3, we have

$$
\begin{aligned}
& \left.\frac{d}{d t} I(t u)\right|_{t=1}=\|u\|^{2}-\int_{\Omega} f(x, u) u d x \\
& =\left(1-\frac{\sigma}{2}\right)\|u\|^{2}+\int_{|u| \leq r}(\sigma F(x, u)-u f(x, u)) d x+\int_{|u|>r}(\sigma F(x, u)-u f(x, u)) d x \\
& \geq\left(1-\frac{\sigma}{2}\right)\|u\|^{2}+(\alpha-\varepsilon) \int_{\Omega} \psi_{1}|u|^{2} d x-c_{\varepsilon} \int_{\Omega} \theta|u|^{p} d x \\
& \geq\left(1-\frac{\sigma}{2}+\frac{\alpha-\varepsilon}{\lambda_{1}\left(\phi, \psi_{1}\right)}\right)\|u\|^{2}-C_{\varepsilon}\|u\|^{p}
\end{aligned}
$$

for some $C_{\varepsilon}>0$. Since $2<p$, then

$$
\left.\frac{d}{d t} I(t u)\right|_{t=1}>0
$$

for $\varepsilon$ small enough and $0<\|u\| \leq \rho$ for $\rho>0$.

Then similarly to the proof of Lemma 2.6 in [8], we have

$$
C_{q}(I ; 0)=0, \text { for all } q \in \mathbb{Z} .
$$

\section{The PROOF OF THE THEOREMS}

Lemma 3.1. Assume $\left(f_{1}\right),\left(f_{3}\right)$ with $\mu=\infty$ and $\left(f_{4}\right)$. Then $C_{q}(I ; \infty) \cong 0$ for all $q \in \mathbb{Z}$.

Proof. Let $S=\{u \in H:\|u\|=1\}$. For any $u \in S$, from $\left(f_{3}\right)$ we have

$$
\begin{aligned}
I(t u) & =\frac{1}{2} t^{2}\|u\|^{2}-\int_{\Omega} F(x, t u) d x \\
& =t^{2}\left[\frac{1}{2}\|u\|^{2}-\int_{\Omega} \frac{F(x, t u)}{(t u)^{2}} u^{2} d x\right] \rightarrow-\infty, \text { as } t \rightarrow \infty .
\end{aligned}
$$

Then there is a constant $A>0$ such that for any $a>A$ there exists $t_{0}$ such that $I\left(t_{0} u\right) \leq-a$. Hence, if

$$
I(t u)=\frac{1}{2} t^{2}\|u\|^{2}-\int_{\Omega} F(x, t u) d x \leq-a,
$$

using $\left(f_{4}\right)$, for any $u \in S$ we have

$$
\begin{aligned}
\frac{d}{d t} I(t u) & =t-\int_{\Omega} f(x, t u) u d x \\
& =\frac{1}{t}\left[t^{2}-\int_{\Omega} 2 F(x, t u) d x+\int_{\Omega} 2 F(x, t u) d x-\int_{\Omega} f(x, t u) t u d x\right] \\
& \leq-\frac{2 a}{t}<0
\end{aligned}
$$

Therefore, for any fixed $a>A$, by the Implicit Function Theorem there exists a unique $T:=T(u)>0$ such that $I(T(u) u)=-a$ for $u \in S$. Then following the argument of Proposition 3.1 in [1], we can deduce that

$$
C_{q}(I ; \infty)=H_{q}\left(H, I^{-a}\right) \cong H_{q}(H, H \backslash\{0\})=0 .
$$


Lemma 3.2 (7], Theorem 4.7). Let $X$ be a Banach space which splits into the direct sum $X=X^{+} \oplus X^{-}$with $X^{-}$finite dimensional. Let $I: X \rightarrow \mathbb{R}$ be continuous, $a, b \in \mathbb{R}$ and $r>0$ be such that

$$
\max _{S_{r}^{-}} I<a<\inf _{X^{+}} I \text { and } \max _{B_{r}^{-}} I<b,
$$

where $B_{r}^{-}=X^{-} \cap \bar{B}_{r}(0), S_{r}^{-}=X^{-} \cap \partial \bar{B}_{r}(0)$. Assume that I satisfies condition (PS) in $I^{-1}([a, b])$. Then $K \cap I^{-1}([a, b]) \neq \emptyset$. More precisely, the following alternative holds: either $K \cap I^{-1}([a, b])$ is an infinite set or there exists $u \in K \cap I^{-1}([a, b])$ such that $C_{n}(I ; u) \neq\{0\}$, where $n=\operatorname{dim} X^{-}$.

Lemma 3.3. Assume $\left(f_{1}\right)$ and $\left(f_{3}\right)$ with $\mu<\infty$. Let $\left\{u_{n}\right\}$ be a sequence in $H$, weakly convergent to a function $u$ and $\rho_{n}>0$ with $\rho_{n} \rightarrow \infty$. Then

$$
\begin{gathered}
\frac{f\left(x, \rho_{n} u_{n}\right)}{\rho_{n}} \rightarrow \mu \psi_{1} u \text { in } L^{1}(\Omega), \\
\frac{F\left(x, \rho_{n} u_{n}\right)}{\rho_{n}^{2}} \rightarrow \frac{1}{2} \mu \psi_{1} u^{2} \text { in } L^{1}(\Omega) .
\end{gathered}
$$

Proof. Since $u_{n} \rightarrow u$ in $H$, then

$$
u_{n} \rightarrow u \text { a.e. } x \in \Omega \text {. }
$$

Then, it is easily seen that $\frac{f\left(x, \rho_{n} u_{n}\right)}{\rho_{n}} \rightarrow \mu \psi_{1} u$ a.e. $x \in \Omega$. From $\left(f_{1}\right)$ and $\left(f_{3}\right)$, there exists a $C>0$ such that

$$
\frac{\left|f\left(x, \rho_{n} u_{n}\right)\right|}{\rho_{n}} \leq C \psi_{1}\left|u_{n}\right| \text { for a.e. } x \in \Omega \text {. }
$$

Since $\psi_{1}(x) u_{n}(x) \rightarrow \psi_{1}(x) u(x)$ strongly in $L^{1}(\Omega)$, from (a variant of) Lebesgue's dominated convergence theorem we deduce that

$$
\frac{f\left(x, \rho_{n} u_{n}\right)}{\rho_{n}} \rightarrow \mu \psi_{1} u \text { in } L^{1}(\Omega)
$$

Similarly, we have

$$
\frac{F\left(x, \rho_{n} u_{n}\right)}{\rho_{n}^{2}} \rightarrow \frac{1}{2} \mu \psi_{1} u^{2} \text { in } L^{1}(\Omega)
$$

Lemma 3.4. Assume $\left(f_{1}\right)$ and $\left(f_{3}\right)$ with $\mu \in\left(\lambda_{m}\left(\phi, \psi_{1}\right), \lambda_{m+1}\left(\phi, \psi_{1}\right)\right)$. Then there is a critical point $u_{0}$ of I with

$$
C_{m}\left(I ; u_{0}\right) \neq 0 .
$$

Proof. Assume $V=\operatorname{span}\left\{u_{1}, u_{2}, \cdots, u_{m}\right\}$, where $u_{i}$ denotes the $i$ th eigenfunction of problem (0.6). Then $H=V \oplus V^{\perp}$. In fact, if $u \in V^{\perp}$, then

$$
\|u\|^{2} \geq \lambda_{m+1}\left(\phi, \psi_{1}\right) \int_{\Omega} \psi_{1} u^{2} d x .
$$

If $u \in V$, then $u=\sum_{i=1}^{m} a_{i} u_{i}$. It is easy to see that

$$
\|u\|^{2}=\sum_{i=1}^{m} a_{i}^{2} \lambda_{i}\left(\phi, \psi_{1}\right) \int_{\Omega} \psi_{1} u_{i}^{2} d x<\lambda_{m}\left(\phi, \psi_{1}\right) \int_{\Omega} \psi_{1} u^{2} d x .
$$

Using Lemma 3.3 and (3.4), for $u \in V$ we have

$$
\liminf _{\|u\| \rightarrow \infty, u \in V} \frac{I(u)}{\|u\|^{2}}=\liminf _{\|u\| \rightarrow \infty, u \in V} \frac{1}{\|u\|^{2}}\left[\frac{1}{2}\|u\|^{2}-\int_{\Omega} F(x, u) d x\right]<0 .
$$


Using Lemma 3.3 and (3.3), for $u \in V^{\perp}$ we have

$$
\liminf _{\|u\| \rightarrow \infty, u \in V^{\perp}} \frac{I(u)}{\|u\|^{2}}=\liminf _{\|u\| \rightarrow \infty, u \in V^{\perp}} \frac{1}{\|u\|^{2}}\left[\frac{1}{2}\|u\|^{2}-\int_{\Omega} F(x, u) d x\right]>0 .
$$

From (3.5), it follows that

$$
\lim _{\|u\| \rightarrow \infty, u \in V} I(u)=-\infty .
$$

On the other hand, from (3.6), we have $\liminf _{u \in V^{\perp}} I(u)>-\infty$. According to Lemma 1.4, we can therefore apply Lemma 3.2 to obtain the existence of a critical point $u_{0}$ of $I$ with $C_{m}\left(I ; u_{0}\right) \neq\{0\}$.

The proof of Theorem 0.1. From Lemma 2.1, we have

$$
C_{q}(I ; 0) \cong \delta_{q, 0} \mathbb{Z}, \text { for all } q \in \mathbb{Z}
$$

Case 1. From Lemma 3.4, we have $C_{m}\left(I ; u_{0}\right) \neq\{0\}$. Since $m>0$ and Lemma 1.4, $u_{0} \neq 0$, and problem $(0.1)$ has at least one nontrivial solution $u_{0} \in H$.

Case 2. From Lemma 3.1, we have

$$
C_{q}(I ; \infty)=0, \text { for all } q \in \mathbb{Z} .
$$

From Lemma $1.5, C_{0}(I ; 0) \neq C_{0}(I ; \infty)$, and problem $(0.1)$ has at least one nontrivial solution.

The proof of Theorem 0.2. From Lemma 2.2, we have

$$
C_{l}(I ; 0) \neq 0 .
$$

Case 1. From Lemma 3.4, we have $C_{m}\left(I ; u_{0}\right) \neq\{0\}$, since $m \neq l$ and given Lemma 1.4. Then $u_{0} \neq 0$ and problem (0.1) has at least one nontrivial solution $u_{0} \in H$.

Case 2. From Lemma 3.1, we have

$$
C_{q}(I ; \infty)=0, \text { for all } q \in \mathbb{Z} .
$$

Then $C_{l}(I ; 0) \neq C_{l}(I ; \infty)$. Using Lemma 1.5 , we can deduce that problem (0.1) has at least one nontrivial solution.

The proof of Theorem 0.3. From Lemma 2.3, we have

$$
C_{q}(I ; 0) \cong 0, \text { for all } q \in \mathbb{Z} .
$$

From Lemma 3.4, we have $C_{m}\left(I ; u_{0}\right) \neq\{0\}$. From Lemma 1.4, $u_{0} \neq 0$, and problem (0.1) has at least one nontrivial solution $u_{0} \in H$.

\section{REFERENCES}

1. Adimurthi, N. Chaudhuri, M. Ramaswamy, An improved Hardy-Sobolev inequality and its application. Proc. Amer. Math. Soc., 130 (2002), 489-505. MR1862130 (2002j:35232)

2. Adimurthi, M.J. Esteban, An improved Hardy-Sobolev inequality in $W^{1, p}$ and its application to Schrödinger operators. Nonlinear Differ. Equ. Appl., 12 (2005), 243-263. MR 2184082 (2006g:26033)

3. Adimurthi, K. Sandeep, Existence and non-existence of the first eigenvalue of the perturbed Hardy-Sobolev operator. Proc. Roy. Soc. Edinburgh Sect. A, 132 (2002), 1021-1043. MR.1938711 (2003i:35210)

4. G. Barbatis, S. Filippas, A. Tertikas, A unified approach to improved $L^{p}$ Hardy inequalities with best constants. Trans. Amer. Math. Soc., 356 (2004), 2169-2196. MR2048514 (2005a:26016) 
5. P. Caldiroli, R. Musina, On a variational degenerate elliptic problem. Nonlinear Differ. Equ. Appl., 7 (2000), 187-199. MR.1771466 (2001g:35090)

6. K. C. Chang, Infinite dimensional Morse theory and multiple solution problems, Progress in Nonlinear Differential Equations and Their Applications. Vol. 6, Birkhäuser, Boston, 1993. MR 1196690 (94e:58023)

7. J-N. Corvellec, Morse theory for continuous functionals. J. Math. Anal. Appl., 196 (1995), 1050-1072. MR1365240 (96m:58037)

8. F. Fang, S.B. Liu, Nontrivial solutions of superlinear p-Laplacian equations. J. Math. Anal. Appl., 351 (2009), 138-146. MR2472927 (2010a:35060)

9. A. Ferrero, F. Gazzola, Existence of solutions for singular critical growth semilinear elliptic equations. J. Differ. Equ., 177 (2001), 494-522. MR1876652(2002m:35068)

10. J.P. Garcia Azorero, I. Peral Alonso, Hardy inequalities and some critical elliptic and parabolic problems. J. Differ. Equ., 144 (1998), 446-476. MR1616905 (99f:35099)

11. Q.S. Jiu, J.B. Su, Existence and multiplicity results for Dirichlet problems with p-Laplacian. J. Math. Anal. Appl., 281 (2003), 587-601. MR1982676 (2004e:35068)

12. J.Q. Liu, The Morse index of a saddle point. Systems Sci. Math. Sci., 2 (1989), 32-39. MR.1110119 (92e:58041)

13. J.Q. Liu, J.B. Su, Remarks on multiple nontrivial solutions for quasi-linear resonant problems. J. Math. Anal. Appl., 258 (2001), 209-222. MR1828101 (2002g:35083)

14. Y.T. Shen, Zh.H. Chen, Nonlinear degenerate elliptic equation with Hardy potential and critical parameter. Nonlinear Anal., 69 (2008), 1462-1477. MR2426707 (2009i:35116)

15. Y.T. Shen, Zh.H. Chen, Sobolev-Hardy space with general weight. J. Math. Anal. Appl., 320 (2006), 675-690. MR2225986 (2007a:46037)

16. Y.T. Shen, X.K. Guo, Weighted Poincaré inequalities on unbounded domains and nonlinear elliptic boundary value problems. Acta Math. Sci. Ser. B Engl. Ed., (3)4 (1984), 277-286. MR812926 (87d:35054)

17. Y.T. Shen, Y.X. Yao, Nonlinear elliptic equations with critical potential and critical parameter. Proc. Roy. Soc. Edinburgh, 136A (2006), 1041-1051. MR2266400 (2008f:35136)

Department of Mathematics, South China University of Technology, Guangzhou 510640, People's Republic of China

E-mail address: ymin.zhang@mail.scut.edu.cn

Department of Mathematics, South China University of Technology, Guangzhou 510640, People's Republic of China

E-mail address: yangjun@scut.edu.cn

Department of Mathematics, South China University of Technology, Guangzhou 510640, People's Republic of China

E-mail address: maytshen@scut.edu.cn 